\title{
Modelling of a Novel High-Impedance Matching Layer for High Frequency (>30 MHz) Ultrasonic Transducers
}

\author{
Y. Qian, N. R. Harris \\ School of Electronics and Computer Science, University of Southampton, University Road, SO17 1BJ \\ Southampton, UK
}

\begin{abstract}
This work describes a new approach to impedance matching for ultrasonic transducers. A single matching layer with high acoustic impedance of 16 MRayls is demonstrated to show a bandwidth of around $70 \%$, compared with conventional single matching layer designs of around $50 \%$. Although as a consequence of this improvement in bandwidth, there is a loss in sensitivity, this is found to be similar to an equivalent double matching layer design. Designs are calculated by using the KLM model and are then verified by FEA simulation, with very good agreement Considering the fabrication difficulties encountered in creating a high-frequency double matched design due to the requirement for materials with specific acoustic impedances, the need to accurately control the thickness of layers, and the relatively narrow bandwidths available for conventional single matched designs, the new approach shows advantages in that alternative (and perhaps more practical) materials become available, and offers a bandwidth close to that of a double layer design with the simplicity of a single layer design. The disadvantage is a trade-off in sensitivity. A typical example of a piezoceramic transducer matched to water can give a $70 \%$ fractional bandwidth (comparable to an ideal double matched design of $72 \%$ ) with a $3 d B$ penalty in insertion loss.
\end{abstract}

\section{Introduction}

Piezoelectric ceramics have been used in many broadband transducers in the field of ultrasonics for several decades [1]. The ultrasonic waves generated by the ceramic layer radiate into a low-impedance 
fluid load (usually water). However, the impedance mismatch between the ceramic and the water medium causes low energy transfer efficiency and a narrow bandwidth. However, it is well known that this efficiency can be improved by inserting single or multiple matching layers to cancel the energy reflection at the interface of the two mediums [2].

For piezoelectric ceramic transducers driving into water, a bandwidth of $40-50 \%$ can be achieved by using conventional single matching layers. Up to $70 \%$ can be achieved by using double matching layers, while applying more matching can in principle further increase the bandwidth [2]. Nevertheless, multiple matching layers are not widely used in current transducers, especially for high frequency (>30MHz) applications, mainly because of fabrication difficulties. These include layer adhesion, thickness accuracy, and selection of applicable materials. With increasing frequency, these issues become more significant, as dimensional tolerances become finer. Although much effort has been expended on the alternative approach of modifying the acoustic impedance of piezoelectric materials to increase the bandwidth, for example, by using piezoelectric composites or polymers [3, 4], there are few publications that address the problem of matching layer design. This is because the standard methods for impedance matching are well defined. However, in this paper, the use of higher impedance materials for single layer matching systems is explored, rather than the traditional method of using impedances between those of the two layers to be matched. Traditionally, impedances lower than 10MRayls would be used in order to match piezoceramics and water, in itself causing issues for material selection. Here, however, we model the use of higher impedances of around 16MRayls with success, allowing more accessible materials to be used, such as certain types of glass. Such an approach offers an improved bandwidth (comparable to double layer matching), with an acceptable loss in sensitivity, offering a good compromise between bandwidth, ease of construction and insertion loss. 
This paper is organised in the following way: In Section II we briefly discuss a typical transducer construction and describe the principle of matching and the use of quarter-wave sections. The paper then describes an alternative approach utilising a combination of electrical and mechanical matching that allows an extension of the transducer bandwidth. Section III illustrates the approach by investigating the design of a $30 \mathrm{MHz}$ PZT based transducer with a single matching layer using classical 1D KLM modelling in order to illustrate the mechanisms at work. This is then verified by FEA analysis, and the comparison of the results discussed, with concluding remarks in Section IV.

\section{Design of high-impedance single matching layer}

Figure 1 illustrates the cross-section of a typical transducer structure, including backing layer, ceramic layer and matching layer(s). The transducer is designed to facilitate the transmission of ultrasound from the ceramic piezoelectric material to the fluid medium (typically water). In principle, the transducer can be made by several different techniques, such as thin-film, machined ceramic, or dice and fill composites, For the purposes of this paper we assume the transducer is a piezoceramic layer, but the matching techniques described are not constrained by this assumption.

In the example shown, the backing layer itself is terminated at an air boundary, and the fluid layer is considered infinite. 


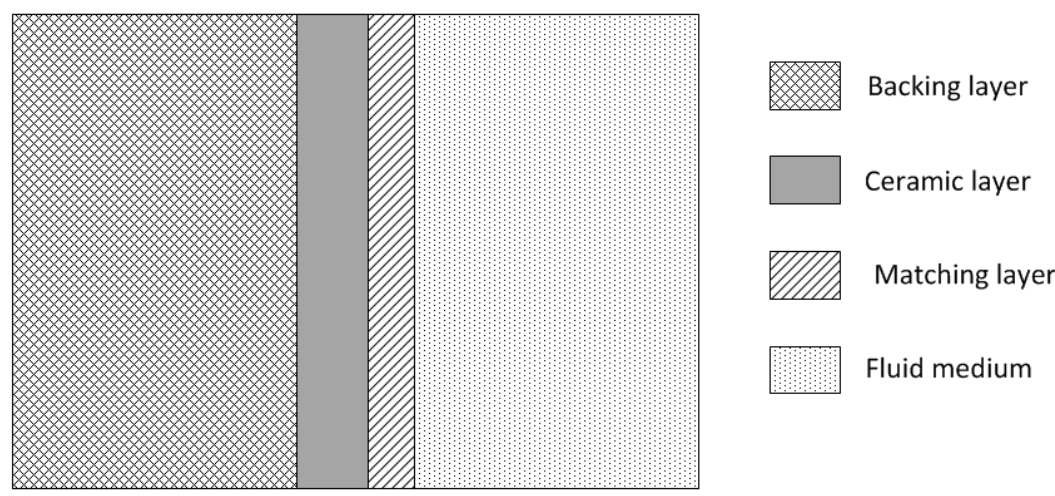

Figure 1 Cross-section of a typical transducer

Since the acoustic impedance of a commonly-used ceramics is around 35MRayls, and the value for water is only 1.5MRayls, this impedance mismatch causes inefficient ultrasonic transmission as in effect the ultrasonic energy cannot escape from the piezoelectric material. To increase the efficiency, matching layer(s) with characteristic thicknesses of $\lambda_{m} / 4\left(\lambda_{m}\right.$ - wavelength of sound in the matching layer at the transducer resonance) are introduced between the piezoelectric ceramic and the fluid medium, whose acoustic impedance follows a conventional design rule expressed below [2],

$Z_{m}=\sqrt{Z_{0} Z_{R}}$

where $Z_{m}, Z_{0}, Z_{R}$ are the acoustic impedances of the matching material, ceramic and fluid load (or water), respectively. Thus the impedance of a single ideal matching layer is about 7MRayls. There are very few useful materials with acoustic impedance near this value, and so compromises have to be made. Epoxies commonly used to form a matching layer have their acoustic impedance in the range of 24MRayls and are sometimes used in transducers. They give some useful improvement in bandwidth compared with having no matching layer, but at a cost in terms of sensitivity compared with an ideal match. To reach the impedance of 7MRayls for ideal matching, heavy particles must be loaded into the epoxy to increase its impedance [5].

However, the ideal matching layer only realises the maximum energy transfer from the ceramic front surface to the fluid medium at the resonance of the ceramic; the energy efficiency reduces as the 
operating frequency moves away from the resonance. This is now discussed in more detail, with a view to explaining how it is possible to improve the bandwidth of single layer designs, and we start by looking into the matching principle. Figure 2 shows an equivalent circuit of the transducer where the quarterwavelength matching layer can be treated as a transmission line and is represented by a transfer matrix $A B C D$. Here the transducer without matching layer is treated as a black box, and $Z_{\text {in }}$ is the input impedance to represent the matching layer and fluid load combined, as seen by the transducer.

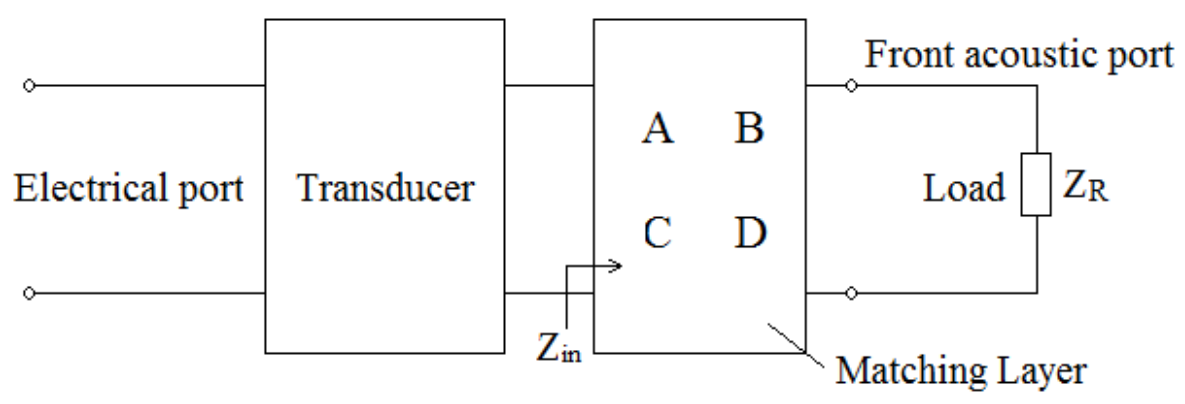

Figure 2 Equivalent circuit of a transducer with matching layer represented by transfer matrix For a transducer without matching layer, the impedance mismatch is the difference between $Z_{0}$ and $Z_{R}$; while for the one with matching layer, it actually becomes the difference between $Z_{0}$ and $Z_{\text {in }}$. If $Z_{\text {in }}=Z_{0}$, the mismatch will be eliminated and therefore no mechanical energy loss will be found in the interface during the transmission, and so it is important to investigate the behaviour of $Z_{\text {in }}$ as the driving frequency moves away from the resonant frequency. The equation describing $Z_{i n}$ is expressed as follows [6],

$Z_{\text {in }}=\frac{A Z_{R}+B}{C Z_{R}+D}$

$\left|\begin{array}{ll}A & B \\ C & D\end{array}\right|=\left|\begin{array}{cc}\cos (k d) & j Z_{m} \sin (k d) \\ j \sin (k d) / Z_{m} & \cos (k d)\end{array}\right|$

where $k$ is the wave number $\left(k=2 \pi / \lambda, \lambda\right.$-wavelength), and $d$ denotes the thickness $\left(d=\lambda_{0} / 4\right)$. Thus the amplitude of $Z_{\text {in }}$ is expressed as, 
$\left|Z_{\text {in }}\right|=\left|\frac{Z_{R} \cos (k d)+j Z_{m} \sin (k d)}{\cos (k d)+j \sin (k d) Z_{m} / Z_{R}}\right|=Z_{m} \cdot \sqrt{\frac{Z_{R}^{2} \cot ^{2}(k d)+Z_{m}^{2}}{Z_{m}^{2} \cot ^{2}(k d)+Z_{R}^{2}}}$

By using the definitions of $k=2 \pi / \lambda$ and $d=\lambda_{0} / 4$, Equation (4) can be further simplified to,

$\left|Z_{\text {in }}\right|=Z_{m} \cdot \sqrt{\frac{Z_{R}^{2} \cot ^{2}(\theta)+Z_{m}^{2}}{Z_{m}^{2} \cot ^{2}(\theta)+Z_{R}^{2}}}$

$\theta=\frac{\pi}{2} \cdot \frac{\lambda_{0}}{\lambda}=\frac{\pi}{2} \cdot \frac{f}{f_{0}}$

where $f$ and $f_{0}$ refers to the driving frequency and resonant frequency, respectively. Here $\vartheta$ is an authordefined parameter related to the transducer bandwidth. The $-6 \mathrm{~dB}$ fractional bandwidth is determined by the lower and higher cut-off frequency $f_{H}, f_{L}$ respectively, with their relations expressed as follows,

$B W=\left(f_{H}-f_{L}\right) / f_{c} \quad ; \quad f_{c}=\left(f_{L}+f_{H}\right) / 2$

where $B W$ denotes the $-6 \mathrm{~dB}$ fractional bandwidth, $f_{C}$ is central frequency and is generally equal to transducer resonant frequency $f_{0}$. By using Equations (6) and (7) and assuming that $f_{C}=f_{0}$, it is found that,

$B W=\frac{2}{\pi}\left(\theta_{H}-\theta_{L}\right) \quad ; \quad \theta_{0}=\frac{\pi}{2}$

where $\vartheta_{0}, \vartheta_{L}$ and $\vartheta_{H}$ are the $\vartheta$ values at $f_{0}, f_{L}$ and $f_{H}$ respectively. Assuming that the fractional bandwidth has a maximum of 1 , this gives a range of values for $\vartheta$ ranging from

$\theta_{L}=\theta_{o}-\frac{\theta_{0}}{2}=\frac{\pi}{2}-\frac{\pi}{4} ; \quad \theta_{H}=\theta_{o}+\frac{\theta_{0}}{2}=\frac{\pi}{2}+\frac{\pi}{4}$

Consequently, the range of the factor " $\cot ^{2}(\vartheta)$ " in Equation (5) is obtained, which varies from 0 for a BW of zero, to 1 for a BW of 1 . Since $Z_{m}$ in most transducer applications is much larger than $Z_{R}$, it is reasonable to assume that $Z_{m}{ }^{2} \gg>Z_{R}^{2}$. Thus the assumption of $Z_{m}{ }^{2} \gg Z_{R}{ }^{2} \cdot \cot ^{2}(\vartheta)$ becomes valid as well. Equation (5) can be simplified to, 
$\left|Z_{\text {in }}\right|=Z_{m}^{2} \cdot \sqrt{\frac{1}{Z_{m}^{2} \cot ^{2}(\theta)+Z_{R}^{2}}}$

At resonance $\left(f=f_{0}\right),\left|Z_{i n}\right|$ reaches a maximum value of $Z_{m}{ }^{2} / Z_{R}$ (also reflected by Equation 1 ). This allows an ideal 7 MRayls single matching layer to make $\left|Z_{\text {in }}\right|$ close to $Z_{0}(\approx 35 \mathrm{MRayls})$, in order to eliminate the impedance mismatch. However, Equation (6) also implies that the mismatch will increase when $f$ moves away from $f_{0}$. Thus an approach is developed to decrease the mismatch away from the band centre, (with a consequential increase in mismatch at the band centre) so that the energy loss away from band centre, especially around the $-6 \mathrm{~dB}$ band edges will be reduced. The bandwidth therefore could be extended.

From Equation (6), it is apparent that $\left|Z_{\text {in }}\right|$ increases as $Z_{m}$ increases. This is true whether at or away from band centre, as illustrated in Figure 3 showing the relation between $\left|Z_{i n}\right|, Z_{m}$ and $f$. Here $f_{0}$ is set to $30 \mathrm{MHz}$ as an example. It can be seen that all the high-impedance matching layers have a $\left|Z_{\text {in }}\right|$ equal to $Z_{0}$ (35MRayls) at 2 frequencies, whereas the ideal match only has 1 frequency (band centre) where this is true. However although the high impedance layers allow a match at 2 points either side of bandcentre, there is obviously a mismatch penalty at band-centre and this will consequently cause a large dip in the insertion loss spectrum. For example, in the case of $Z_{m}=10$ MRayls, $Z_{\text {in }}$ has a maximum of about 70 MRayls at $30 \mathrm{MHz}$, which is very different from the matched case of 35 MRayls, but has a $Z_{\text {in. }}$ of 35 MRayls at frequencies of about $25 \mathrm{MHz}$ and $35 \mathrm{MHz}$. 


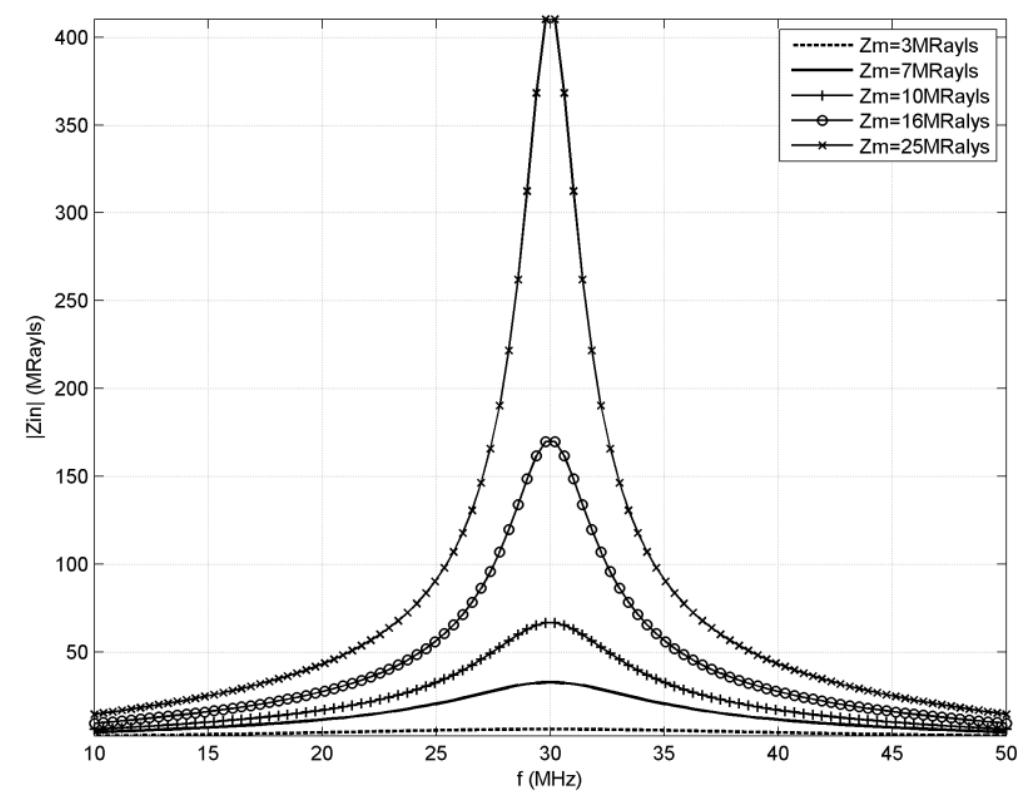

Figure 3 The relationship between $\left|Z_{i n}\right|, Z_{m}$

At this point it should be remembered that impedance matching only optimizes the efficiency of mechanical energy transfer between the transduction element and the target medium [2]. It doesn't maximise the efficiency of the transducer system as a whole. The match of the electrical interface to the transducer also needs to be considered, and this can be optimised by electrical tuning. With a welldesigned tuning network, the loss in mechanical energy due to a high impedance matching layer can be compensated at band centre by the electrical tuning network, potentially reducing the effective mismatch at bandcentre; meanwhile, the decreased mismatch away from band centre is still valid, and these two factors combined can act to increase the total bandwidth. Figure 4 shows a typical tuning network consisting of a series resistor $R_{S}$ and inductor $L_{S}$, where the transducer is represented by a series resistor $R_{A}$, reactance $X_{A}$ and the clamped capacitance $C_{0}[2]$. 


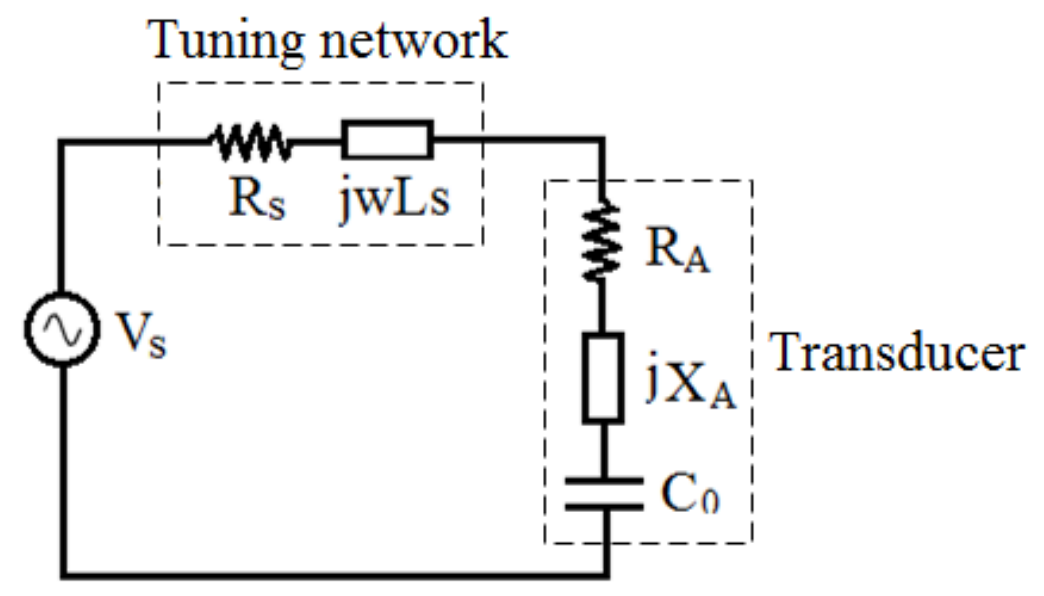

Figure 4 The schematic diagram of a tuning network inserted into a transducer

The inductor $L_{S}$ is designed to cancel the capacitance $C_{0}$ of the transducer, and $R_{S}$ represents the internal resistor of a voltage source $V_{S}$. The transducer resistor $R_{A}$ is required to be equal to $R_{S}(\approx 50 \Omega$ for a standard circuit) at resonance so that maximum power transfer can be achieved. However, $R_{A}$ is a transducer dependent parameter and is not always $50 \Omega$. Instead of tuning $R_{A}$ by changing the transducer design, we simply adjust $R_{\mathrm{S}}$ here for the design convenience, and this is investigated in the next section.

\section{Performance of high-impedance single matching layer}

An example high frequency $(30 \mathrm{MHz})$ transducer is analyzed here to show the influence of highimpedance single matching layer, since these have more fabrication issues in depositing the matching layer(s) compared to lower frequency transducers, although the same principles apply. As a comparison, the reader is referred to Lukac et al's paper [14] where a comprehensive and detailed design of a $30 \mathrm{MHz}$ array is described. Developments of this array are currently being used in high performance imaging systems [15], however, the array is a key part of these. The referred to array is a double matching layer design, but the paper does not give many details about the actual matching layer construction, other than indicating that the matching layer impedances are around 8 and 3 MRayls, and that it is necessary 
to lap the individual layers to the correct thickness, which is a time -consuming process. The new matching techniques described here may well offer an alternative in such systems as alternative methods such as spin coating may be applicable, or at the very least, only one layer of matching needs to be prepared. The bandwidth in Lukac's paper is given as $70 \%$ with no lens, which compares with the calculations in this paper where a bandwidth of $70 \%$ is predicted for a high impedance match although an exact direct comparison is difficult to establish. It is worth noting that another of the difficulties associated with high-frequency arrays centres around the creation of kerfs, (the gaps between elements) as these become significant as the dimensions of the elements falls. In this paper we use a material called PZ34. To provide context for the choice of this material, the authors have investigated screenprinting as a technique for depositing PZT based arrays [16], and the use of less common piezoelectric materials such as PZ34 offer the potential to create kerfless arrays. PZ34 (sourced from Ferroperm) is a grade of lead titanate recommended for high frequency medical transducers as due to its large electromechanical anisotrophy it is potentially more resistant to cross-coupling than other common piezoelectric materials. The matching techniques described in this paper are potentially amenable to deposition by screen printing or spin-coating, as different printable dielectric glasses are available.

To illustrate the matching system, a simple transducer will be modelled. A disk-shaped high frequency transducer usually has its diameter in the range of 2 to $6 \mathrm{~mm}$ [7-9], and thus the transducer here is set to be a $2 \mathrm{~mm}$-diameter aperture. The ceramic transducer layer is $75 \mu \mathrm{m}$ to give a resonant frequency around $30 \mathrm{MHz}$ if PZ34 (provided by Ferroperm) is used. The backing layer is set to have an acoustic impedance of 10 MRayls to reduce transducer ringing.

Once the transducer size and materials are defined, the transducer parameters $R_{A}, X_{A}$ and $C_{0}$ can be calculated. The detailed equations to derive these constants can be found in reference [2]. Consequently, 
the electrical tuning parameter $L_{S}$ is determined to eliminate $C_{0}$, while the resistor $R_{S}$ is allowed to vary to show the influence of electrical tuning.

The 1D KLM model $[10,11]$ is used initially to numerically evaluate the transducer performance by calculating the insertion loss (IL) spectrums. The KLM modelling approach is used in preference to the Mason approach because we have experience in using it and both models have been shown to be equivalent in the literature [17]. Figure 5(a)-(c) then illustrates the relation between $R_{s}$ and IL parameters including bandwidth $B W$, central frequency $f_{c}$ and peak IL ( $I L_{\max }$, to reflect sensitivity), for the transducer with different matching layer designs, respectively. A single matching layer of $Z_{m}=3 \mathrm{MRayls}$ is used to represent common epoxies used in real transducers, a $Z_{m}$ of $7 \mathrm{MRayls}$ is indicative of traditional perfect matching; $Z_{m}$ of 10,16 and 25 MRayls refers to example high-impedance single matching layers. The response for a double matched solution is also displayed for comparison, of which the design follows a so-called maximally flat approach detailed in reference [2]. This approach aims at showing the flattest band.
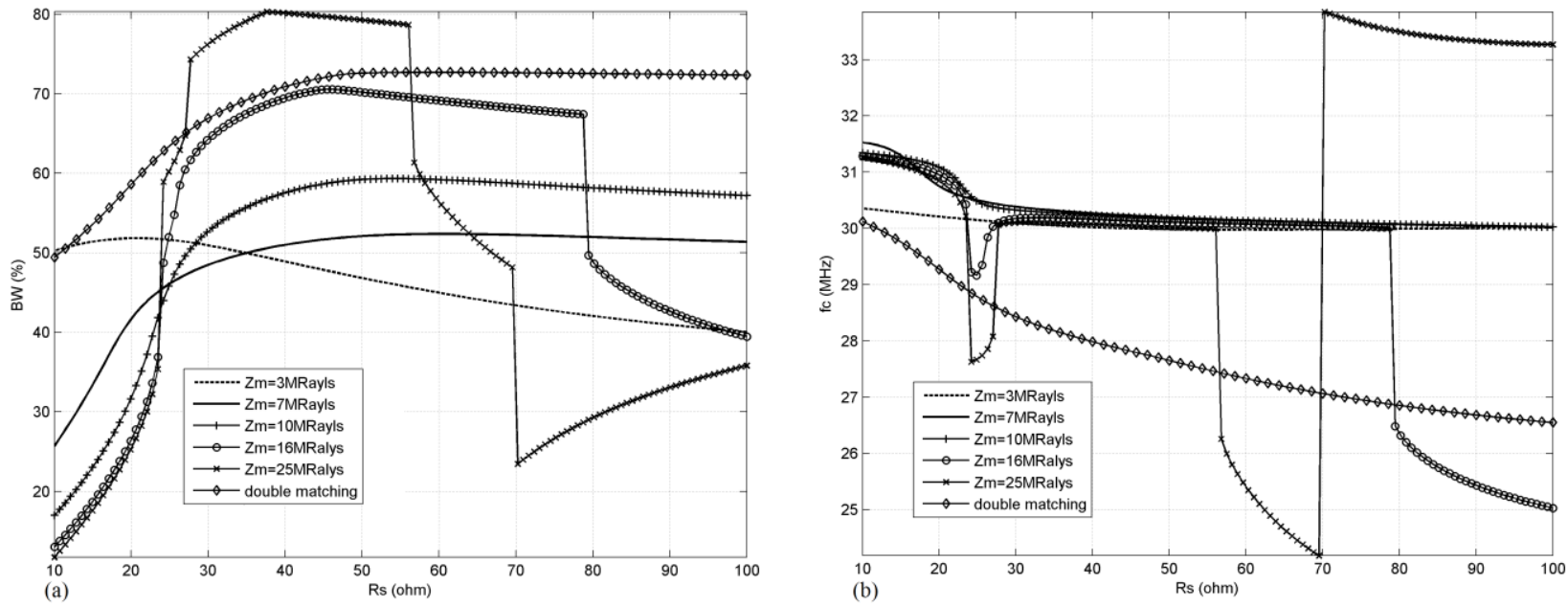

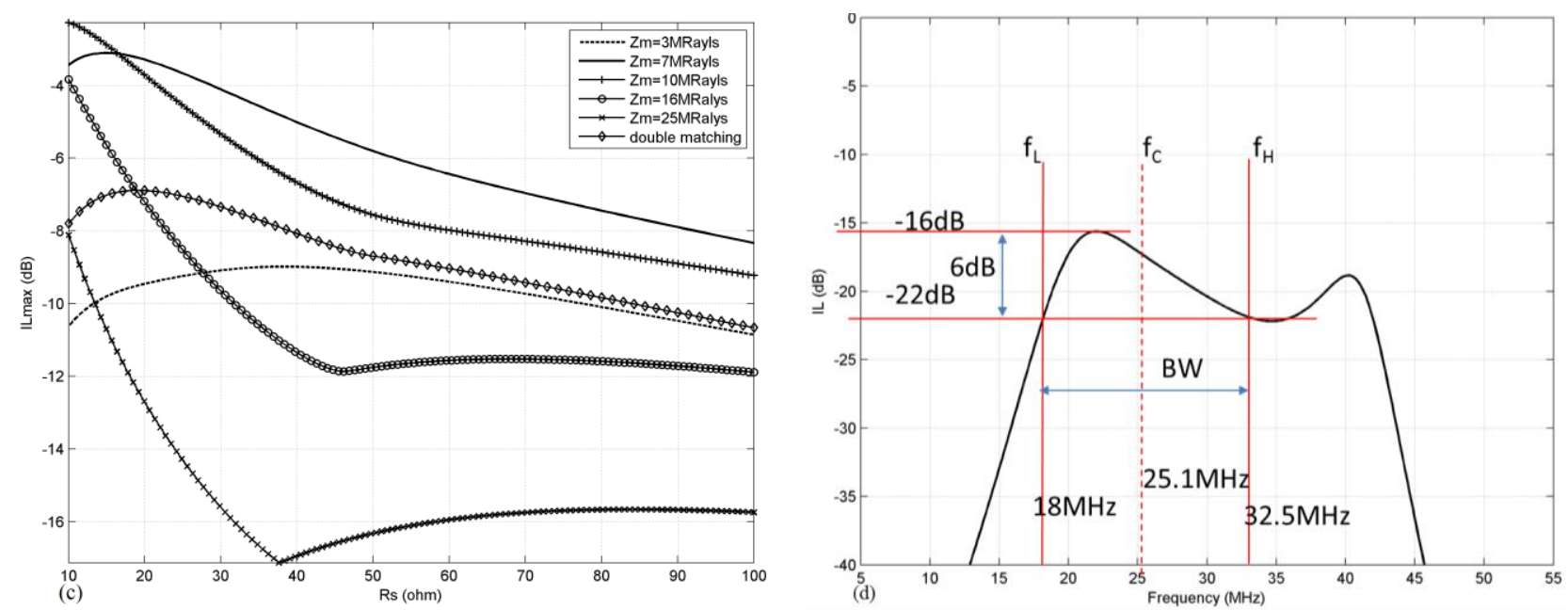

Figure 5 The relation between $R_{s}$ and IL parameters including (a) bandwidth, (b) IL peak and (c) central frequency. (d) shows an example IL frequency response of the transducer using $Z_{m}=25 \mathrm{MRayls}, R_{s}$ is set to be a high value of $60 \Omega$

The responses generated by using a single matching layer are discussed initially. Except for the response of $Z_{m}=3$ MRayls, it can be seen in Figure 5(a) that the maximum bandwidth increases with increasing impedance $Z_{m}$, (about $52 \%$ for $7 \mathrm{MRayl}$, rising to $80 \%$ for $25 \mathrm{MRayl}$ ), and the value of $R_{S}$ for which this maximum bandwidth occurs shows a trend to decrease with increasing $Z_{m}$. More importantly, the range of values for $R_{S}$ that allow these bandwidths reduces as $Z_{m}$ increases. For example, $R_{S}$ can be in the range of $45 \Omega$ to in excess of $100 \Omega$ for $Z_{m}=7$ MRayls, but has to be in the range $38 \Omega$ to $58 \Omega$ for a high bandwidth for $Z_{m}=25 \mathrm{MRayls}$. This is due to the impedance mismatch caused by the larger $Z_{m}$ at resonance, as illustrated in Figure 3 previously. Thus it can be seen that it is important to achieve very good electrical tuning in order to compensate for the large loss in mechanical energy, and this leads to a very narrow $R_{S}$ range suitable for wide band operation. If the electrical tuning is not well-optimized, the electrical tuning is not strong enough to compensate for the mechanical loss, and a dip could still appear at the centre of the IL spectrum. 
As a result, the plot in Figure 5(a) also shows significant abrupt changes in $B W$ value for the higher values (16 and 25 MRayls) of $Z_{m}$. The expected valley at band centre tends to lead to a double-humped IL response, as given in Figure 5(d) as an example. When the valley to peak value becomes large enough, only one of the peaks will be available for bandwidth calculation, resulting in an abrupt reduction in apparent bandwidth. For example, in Figure 5(d), the peak insertion loss has a value of $-16 \mathrm{~dB}$ at $22 \mathrm{MHz}$, and the band can be seen to range from $18 \mathrm{MHz}$ to $32.5 \mathrm{MHz}$, in agreement with Figure $5(\mathrm{a})$ for $Z_{m}$ $=25 \mathrm{MRayls}$ and $R_{s}=60 \Omega$. From consideration of Figure 6 it is apparent that if the peak insertion loss was a fraction of a dB higher, then the second peak centred at $40 \mathrm{MHz}$ in the plot would be included in the bandwidth calculation. It is this type of feature that leads to the abrupt changes visible in Figure 5(a). It is also apparent why the centre frequency has changed in Figure 5(b), as the measurement of bandwidth has become focussed on the first peak. Thus the numerical programme used to obtain these figures takes either the left and right band peak when electrical tuning is not optimized.

The discontinuous spectrums in Figure $5(a)$ and (b) lead to the conclusion that although higher bandwidths are possible, the tuning resistor $R_{s}$ becomes less flexible as higher values of $Z_{m}$ are used. Nevertheless, this is a minor shortcoming requiring only some constraints on the design of the electrical tuning network. Furthermore, as shown in Figure 5(c), the insertion loss is generally adversely affected with increasing $Z_{m}$, since there is an increasing impedance mismatch at the band centre as $Z_{m}$ is increased. Therefore, a very high $Z_{m}$ design is not recommended.

With reference to the double matching layer solution, also shown in Figure 5, as expected it shows an improvement over the single ideal match solution, with the bandwidth improving from $55 \%$ to $73 \%$. However, the response is relatively insensitive to the value of $R_{s}$. Its $I L_{\max }$ is found to be slightly lower than for $Z_{m}=7$ MRayls, while its $f_{c}$ is always smaller than the designed value of $30 \mathrm{MHz}$ and keeps 
decreasing as $R_{s}$ increases. Table 1 lists the maximum value of $B W$ with its corresponding $R_{s}, f_{c}$ and $I L_{\max }$ for all the matching designs in Figure 5.

Table 1 Maximum $B W$ with corresponding $R_{s}, f_{c}$ and $I L_{\max }$ by using different matching layers

\begin{tabular}{ccccccc}
\hline $\boldsymbol{Z}_{\boldsymbol{m}}$ (MRayls) & $\mathbf{3}$ & $\mathbf{7}$ & $\mathbf{1 0}$ & $\mathbf{1 6}$ & $\mathbf{2 5}$ & $\begin{array}{c}\text { Double } \\
\text { Matching }\end{array}$ \\
\hline $\boldsymbol{B W}(\boldsymbol{\%})$ & 51.9 & 52.4 & 59.3 & 70.6 & 80.3 & 72.7 \\
$\boldsymbol{R}_{\boldsymbol{s}}(\boldsymbol{\Omega})$ & 20.5 & 58.9 & 53.9 & 46.1 & 37.6 & 52.3 \\
$\boldsymbol{f}_{c}(\mathrm{MHz})$ & 30.2 & 30.2 & 30.2 & 30.1 & 30.1 & 27.6 \\
$\boldsymbol{I L}_{\max }(\mathrm{dB})$ & -9.43 & -6.37 & -7.76 & -11.7 & -17.1 & -8.78 \\
\hline
\end{tabular}

It is found that a single matching layer with $Z_{m}=16$ MRayls shows a comparable $B W$ to the double matching layer, and with only a $-3 \mathrm{~dB}$ penalty in $I L_{\max }$ or sensitivity. A $25 \mathrm{MRayls}$ single matching layer can give a $B W$ of up to $80 \%$, but insertion losses become very significant, being $8.3 \mathrm{~dB}$ worse than the double match. In addition, all the single matching options have their central frequencies closer to the designed value of $30 \mathrm{MHz}$ than the double matching solution. Comparing $Z_{m}=16 \mathrm{MRayls}$ to the conventional single matching $\left(Z_{m}=3\right.$ and 7 MRayls), a large increase in bandwidth is found for $Z_{m}=16$ MRayls (80.3\% c.w. $51.9 \%$ and $52.4 \%$ ). A slight loss in sensitivity of $2.3 \mathrm{~dB}$ can be seen when compared to $Z_{m}=3$ MRayls (a practically used solution), while it becomes larger $(5.3 \mathrm{~dB})$ when compared to $Z_{m}=7$ MRayls. Nevertheless, it can be claimed that single matching of $Z_{m}=16$ MRayls is a good approximation to double matching; and also shows bandwidth advantages over conventional single matching, especially for $Z_{m}=3$ MRayls.

To the authors' knowledge, materials with $Z_{m}>10$ MRayls haven't been used as single matching layers so far. To show the potential effectiveness of high-impedance matching $\left(Z_{m}=16 \mathrm{MRayls}\right)$ for real transducers, an FEA (Finite Element Analysis) software package (ANSYS 11.0) has been used to simulate transducer behaviour, including the responses of the transducers with $Z_{m}$ of 3,7 and 16 MRayls as well as the double matching. We have experience of ANSYS, which is a well-established general FEA package that is capable of multi-physics simulation. A mesh study was carried out using elements as small as $\lambda / 20$, and a 
mesh size of $\lambda / 8$ was found to give good results. The materials used for all these matching designs are listed in Table 2, where $\rho, c, Z$ and $\alpha$ represent density, longitudinal sound speed, acoustic impedance, and attenuation coefficient respectively.

Table 2 Parameters of the materials for the 4 matching options

\begin{tabular}{|c|c|c|c|c|c|c|c|}
\hline \multicolumn{2}{|c|}{ Matching Layers } & Material & Ref. & $\begin{array}{c}\rho \\
\left(a / \mathrm{cm}^{3}\right)\end{array}$ & $\begin{array}{c}c \\
(\mathrm{~m} / \mathrm{s})\end{array}$ & $\underset{\text { (MRayls) }}{Z}$ & $\begin{array}{c}\alpha(\mathrm{dB} / \mathrm{mm} \\
\left.\cdot \mathrm{MHz}^{-1}\right)\end{array}$ \\
\hline \multirow{3}{*}{$\begin{array}{c}\text { Single } \\
\text { matching }\end{array}$} & option 1 & EPO-TEK 301-2 & [12] & 1.15 & 2650 & 3.05 & 0.32 \\
\hline & option 2 & $\begin{array}{l}\text { 38\% Alumina-loaded } \\
\text { EPO-TEK 301-2 }\end{array}$ & [12] & 2.22 & 3140 & 6.97 & $\approx 0.4$ \\
\hline & option 3 & Glass-flint & ONDA & 3.60 & 4500 & 16.0 & Very low \\
\hline \multirow{2}{*}{$\begin{array}{l}\text { Double } \\
\text { matching }\end{array}$} & layer 1 & $\begin{array}{c}\text { 26\% Tungsten-loaded } \\
\text { EPO-TEK 301-2 }\end{array}$ & [12] & 5.90 & 1550 & 9.15 & $\approx 0.8$ \\
\hline & layer 2 & $\begin{array}{c}\text { 17.5\% Alumina-loaded } \\
\text { EPO-TEK 301-2 }\end{array}$ & [12] & 1.64 & 2620 & 4.30 & $\approx 0.5$ \\
\hline
\end{tabular}

*ONDA: ONDA Corporation, http://www.ondacorp.com/

A commonly available epoxy, EPO-TEK 301-2, is loaded with different heavy particles such as alumina and tungsten to achieve the required acoustic impedances below 10MRayls. Here the parameters of these loaded epoxies are evaluated by the Devaney model [13], which is a method for calculating elastic constants and, by extension the density and velocities of a material consisting of homogeneous and isotropic inclusions within an elastic medium. For the high impedance matching layer, a glass material is found that has an acoustic impedance of 16MRayls (Table 2). 


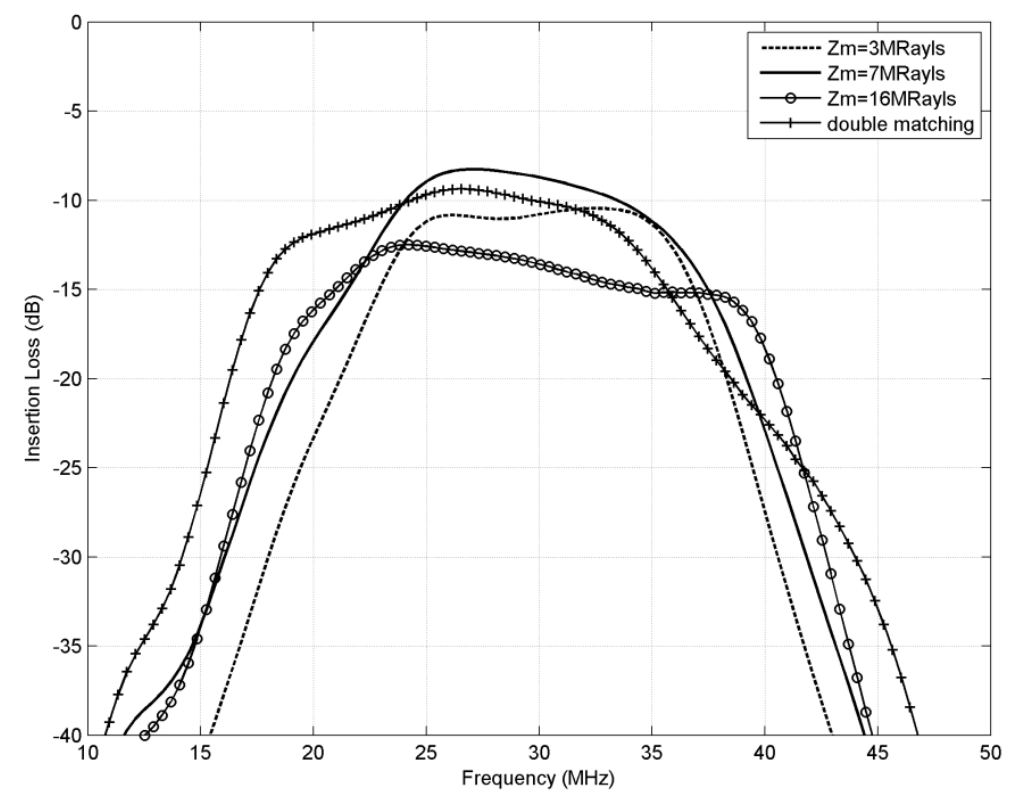

Figure 6 IL spectrums of the transducers using the 4 matching options

Table 3 Parameters including IL peak $I L_{\max }$, central frequency $f_{c}$ and bandwidth $B W$

\begin{tabular}{cccc}
\hline Matching Layer & $\boldsymbol{I L}_{\max }(\mathbf{d B})$ & $\boldsymbol{f}_{c}(\mathbf{M H z})$ & $\boldsymbol{B W}(\%)$ \\
\hline $\boldsymbol{Z}_{m}=$ MMRayls & -10.4 & 29.9 & 50.0 \\
$\boldsymbol{Z}_{m}=\mathbf{7 M R a y l s}$ & -8.26 & 29.5 & 51.2 \\
$\boldsymbol{Z}_{m}=$ 16MRayls & -12.5 & 29.4 & 72.7 \\
Double matching & -9.35 & 26.7 & 68.8 \\
\hline
\end{tabular}

By using the FEA method, the IL spectrums of the transducers using the 4 matching designs are illustrated in figure 6, with their band parameters listed in Table 3. All the FEA results for these matching designs agree well with the KLM-model results listed in Table 2, except for slightly lower $I L_{\max }$ values for the FEA responses. For example, at $25 \mathrm{MRayls}$, the difference is $0.8 \mathrm{~dB}$. This loss might be due to the lateral energy dissipation in FEA, which is not evaluated in the numerical KLM model. Nevertheless, the FEA results suggests that the high-impedance single matching layer (in this case 16M Rayls) is able to show a very broad-band response (73\%) compared to the others $(50-51 \%)$, while the cost in sensitivity generally ranges from -3 to $-4 \mathrm{~dB}$ (Figure 6). 


\section{Conclusion}

This paper details a theoretical investigation into an alternative method for matching ultrasonic transducers, by considering the use of higher value impedance materials. Such materials are attractive because they are more readily available than materials for lower impedance matching. By consideration of both the mechanical and electrical matching requirements, it is possible to create single layer matching systems with higher impedance materials that offer a good compromise between insertion loss and bandwidth, with performance that compares well to double matched systems. Thus it is found as an example that a single matching layer with acoustic impedance of $16 \mathrm{MRayls}$ shows a bandwidth up to $70 \%$; a value comparable to double matching, and at a relatively low cost in sensitivity reduction (about 3dB). These results are analysed using the classical 1D KLM model to gain an insight into the mechanisms at work, and are verified by ANSYS finite element modelling. To conclude, both the numerical and FEA results suggest that high-impedance matching is a potential replacement for current conventional single matching designs requiring a larger bandwidth without going to the complexity of a double layer design.

\section{References}

1. Kossoff, G., The Effects of Backing and Matching on the Performance of Piezoelectric Ceramic Transducers. IEEE Transactions on Sonics and Ultrasonics, 1966. 13(1): p. 20-30.

2. Szabo, T.L., Diagnostic Ultrasound Imaging: Inside Out. 2004: Elsevier Academic Press. pp100117

3. Brown, L.F., Design considerations for piezoelectric polymer ultrasound transducers. IEEE Transactions on Ultrasonics Ferroelectrics and Frequency Control, 2000. 47(6): p. 1377-1396.

4. Smith, W.A. The role of piezocomposites in ultrasonic transducers. IEEE Proc. Ultrasonics Symposium, 1989. pp755-766

5. Webster, R.A., et al. Passive Materials for High Frequency Ultrasound Components. in IEEE Proc. Ultrasonics Symposium, 2007. pp1925-1928 
6. Lockwood, G.R. and Foster, F.S., Modeling and Optimization of High-frequency Ultrasound Transducers. IEEE Transactions on Ultrasonics, Ferroelectrics and Frequency Control, 1994 41(2), pp. 225-230.

7. Foster, F.S., et al., Principles and Applications of Ultrasound Backscatter Microscopy. IEEE Transactions on Ultrasonics, Ferroelectrics and Frequency Control, 1993. 40(5) pp. 608-617.

8. Morton, C.E. and Lockwood, G.R., Design of a $40 \mathrm{MHz}$ Annular Array, IEEE Proc. Ultrasonics Symposium, 2001, pp1135-1138

9. Cannata, J.M., et al., Design of efficient, broadband single-element (20-80 MHz) ultrasonic transducers for medical imaging applications. IEEE Transactions on Ultrasonics Ferroelectrics and Frequency Control, 2003. 50(11), pp. 1548-1557.

10. Krimholtz, R., Leedom, D. A., and G.L. Matthaei, G.L., New equivalent circuits for elementary piezoelectric transducers. Electronics Letters, 1970. 6(13), pp. 398-399.

11. Selfridge, A.R. and Gehlbach, S., KLM Transducer Model Implementation Using Transfer Matrices. IEEE Proc. Ultrasonics Symposium. 1985.pp875-877

12. Wang, H.F., et al., High frequency Properties of Passive Materials for Ultrasonic Transducers. IEEE Transactions on Ultrasonics Ferroelectrics and Frequency Control, 2001. 48(1), pp. 78-84.

13. Devaney, A.J. and H. Levine, Effective elastic parameters of random composites. Applied Physics Letters, 1980. 37(4), pp. 377-379.

14. Lukacs, M, et. al., Performance and Characterisation of New Micromachined High-Frequency Linear Arrays, Transactions on Ultrasonics Ferroelectrics and Frequency Control, 2006, 53(10), pp1719-1729

15 Foster F, et. al., A New 15-50 MHz Array-based Micro-Ultrasound Scanner for Preclinical Imaging Ultrasound in Med. \& Biol.,2009 , 35(10), pp1700-1708

16 Qian, Y., Harris, N.R., Beeby ,S.P., Design of a Novel High Frequency Ultrasound Annular Array Procedia Chemistry 2009, 1, pp413-416

17 Sherrit, $\mathrm{S}$ et al., Comparison of the Mason and KLM Equivalent Circuits for Piezoelectric Resonators in the Thickness Mode. IEEE Proc. Ultrasonics Symposium. 1999.pp921-926 